\title{
Identification of etiologic agents and clinical characteristics for patients suspected of having pertussis in a large Children's Hospital in China
}

\author{
Yue Tao ${ }^{1, \#}$, Mingyu Tang ${ }^{3 \#}$, Lijuan Luo ${ }^{1 \#}$, Long Xiang ${ }^{4}$, Yijun $\mathrm{Xia}^{5}$, Biru $\mathrm{Li}^{4}, \mathrm{Qing}^{\mathrm{Cao}}{ }^{1}, \mathrm{Xi} \mathrm{Mo}^{2}$ \\ ${ }^{1}$ Department of Infectious Diseases, Shanghai Children's Medical Center, Shanghai Jiao Tong University School of Medicine, Shanghai 200127, \\ China; ${ }^{2}$ The Laboratory of Pediatric Infectious Diseases, Pediatric Translational Medicine Institute, Shanghai Children's Medical Center, Shanghai \\ Jiao Tong University School of Medicine, Shanghai 200127, China; ${ }^{3}$ Department of Respiratory Medicine, ${ }^{4}$ Pediatric Intensive Care Unit, Shanghai \\ Children's Medical Center, Shanghai Jiao Tong University School of Medicine, Shanghai 200127, China; ${ }^{5}$ Medical Affairs Director, Great China I \\ bioMérieux (Shanghai) Company, Limited, Shanghai 200127, China \\ Contributions: (I) Conception and design: Y Tao, M Tang, L Luo, Q Cao, X Mo; (II) Administrative support: Q Cao, X Mo; (III) Provision of study \\ materials or patients: M Tang, L Luo, L Xiang, B Li, Q Cao; (IV) Collection and assembly of data: Y Tao, M Tang, L Luo, Q Cao, X Mo; (V) Data \\ analysis and interpretation: Y Tao, M Tang, Y Xia; (VI) Manuscript writing: All authors; (VII) Final approval of manuscript: All authors. \\ \#These authors contributed equally to this work. \\ Correspondence to: Xi Mo; Qing Cao. 1678 Dongfang Rd, Shanghai Children's Medical Center, Shanghai 200127, China. \\ Email: xi.mo@shsmu.edu.cn; caoqing@scmc.com.cn.
}

Background: In China, pertussis is a major health problem with an increasing incidence despite immunization efforts. Timely and accurate diagnosis is essential for the optimal management of pertussis, especially in severe cases.

Methods: Nasopharyngeal swabs or sputum specimens were obtained from patients suspected of having pertussis on the day of hospitalization at Shanghai Children's Medical Center from December 01, 2016, to November 30, 2017. The specimens were tested with the FilmArray Respiratory Panel, a multiplex polymerase chain reaction (PCR) assay that detects 16 viruses, Bordetella pertussis (B. pertussis), Mycoplasma pneumoniae (M. pneumoniae), and Chlamydophila pneumoniae (C. pneumoniae).

Results: Among the 140 children studied, 50.0\% (70/140) were detected with a single pathogen, $45.0 \%$ $(63 / 140)$ were detected with multiple pathogens, and 5.0\% (7/140) had no detected pathogens. Forty-nine $(35 \%, 49 / 140)$ patients tested positive for B. pertussis. Respiratory syncytial virus (RSV), parainfluenza virus (Para) and rhinovirus/enterovirus (Rhino/Entero) were the most prevalent pathogens in patients with pertussis-like syndrome. No significant differences between the groups with pertussis and pertussis-like syndrome were observed regarding the clinical symptoms. Severe cases were more frequently observed in unvaccinated, premature and pertussis/RSV co-infection patients.

Conclusions: Our study highlights the importance of the timely and accurate diagnosis of pertussis based on both clinical symptoms and laboratory methods.

Keywords: B. pertussis; pertussis-like syndrome; pertussis/virus co-infections; FilmArray Respiratory Panel; children

Submitted Mar 27, 2019. Accepted for publication Aug 15, 2019.

doi: 10.21037/atm.2019.08.85

View this article at: http://dx.doi.org/10.21037/atm.2019.08.85 


\section{Introduction}

Bordetella pertussis (B. pertussis) is a fastidious gramnegative coccobacillus bacterium that causes pertussis (whooping cough), a highly contagious human respiratory infection that most often affects very young, unimmunized or incompletely immunized infants (1). Despite the high childhood vaccination rate $(2,3)$, pertussis remains one of the top ten fatal infections in children (4). The typical clinical symptoms of pertussis include repeated paroxysmal cough, an inspiratory whoop and post-cough vomiting (5). The classic presentation of this disease, which is characterized by three phases (catarrhal, paroxysmal and convalescent), is well-known, but has been observed less often since the initiation of immunization (6).

As a vaccine-preventable disease, a resurgence of pertussis in the post-vaccination era has been reported in developed countries despite high vaccination coverage (7-9). A shift in cases from school-age children to adolescents, adults and infants under 1 year of age has been described in the last decade, and the mortality rates in infants have not decreased (10). In China, pertussis is a major health problem, with increasing incidence despite immunization efforts. An abrupt increase in incidence was reported in 2013, 2014 and 2015 , with rates of $0.13 / 100,000,0.25 / 100,000$ and $0.49 / 100,000$, respectively, according to the official data on this infectious disease reported by Chinese Center for Disease Control and Prevention (CDC). Nonetheless, a number of epidemiological studies have suggested that the incidence of pertussis in China was significantly underestimated $(11,12)$. Currently, the timely and accurate diagnosis of pertussis is an urgent need for two reasons: to isolate patients in the early stage of the disease to prevent transmission and to treat patients in a timely manner with macrolide antibiotics to improve patient care.

Although classic pertussis can be reliably diagnosed based on clinical symptoms, the recurrence and atypical manifestations of pertussis highlight the importance of standardized, sensitive and specific laboratory approaches to supplement clinical diagnosis (13). The standard laboratory diagnostic criteria for the identification of $B$. pertussis include culture, polymerase chain reaction (PCR) assays and serodiagnosis (13-15). The culture of nasopharyngeal secretions is currently the gold standard for pertussis diagnosis. However, given the harsh growth conditions and the high standards for samples, the detection rate of culture remains low, and this method is also time consuming (3-7 d). Serological diagnosis needs two specimens from both the acute and recovery periods, limiting its application in early diagnosis. Multiple studies have shown that PCR is the most sensitive approach and should always be included, independent of the stage of the disease, to complement culture in the early stage and serology in the later stage (16-19). Therefore, both the CDC and WHO use positive PCR results as the criteria for pertussis diagnosis $(20,21)$. Despite the fact that the laboratory diagnosis of pertussis in China is based on culture and serology results, these two methods are not routinely available in most Chinese hospitals $(22,23)$. This observation may be the main cause for the underestimation of the pertussis incidence in China.

In addition to the lack of laboratory diagnosis of pertussis, previous studies have shown that the clinical presentation of pertussis is indistinguishable from that of viral respiratory infections (24-26). In addition, coinfections with respiratory viruses such as respiratory syncytial virus (RSV), rhinovirus/enterovirus (Rhino/ Entero) and adenovirus (Adv) frequently occur in infants with pertussis infections $(27,28)$. The current strategy in both laboratory and commercial assays is the development of multiplex PCR platforms for the detection of multiple respiratory pathogens, including B. pertussis (29-31). FilmArray (BioFire Diagnostics, Utah, USA, owned by bioMérieux) is a small, desktop, fully automated multiplex PCR device. The FilmArray Respiratory Panel (FilmArray $\mathrm{RP}, \mathrm{v} 1.7$ ) is able to detect 16 viral and 3 atypical respiratory pathogens, including $B$. pertussis, in a single test, which requires only $5 \mathrm{~min}$ of hands-on time and $65 \mathrm{~min}$ of instrument time (32). The multiplexed nature of this test enables the rapid differentiation of $B$. pertussis from other viruses that also cause pertussis-like syndrome, such as RSV, Adv and parainfluenza 3 (Para 3) (33-35). In addition, this method provides co-infection information for pertussisinfected patients, which may have underlying clinical significance.

According to statistics from 2018, Shanghai has a total population of 24 million people, which includes an urban population of 21 million individuals and a rural population of 3 million. The total GDP is $¥ 3,267.9$ billion and the per capita GDP is $¥ 135,000$. The disposable personal income of the urban population is $¥ 68,000$, and that of the rural population is $¥ 30,000$. Currently, there are 5,298 health care institutions in Shanghai, which includes 364 hospitals and 206,500 health professionals. Although the socio-economic data may provide better understanding of potential bearing on the prevalence of pertussis, the surveillance data on pertussis in Shanghai are limited. In 
2010, a multicenter clinical study enrolled 1,001 patients with a pertussis cough (cough for more than 2 weeks) in Shanghai, Chongqing, Kunming and Yinchuan. The total infection rate in Shanghai was $16.7 \%$ (65/402), which was higher than that in the other regions (36). In 2017, 258 pertussis cases, which were confirmed by PCR and/or culture, were reported by 6 sentinel hospitals in Shanghai (including Shanghai Children's Medical Center, SCMC) according to data from the local CDC (37).

In our previous work, 775 eligible patients with respiratory tract infections (RTIs) were subject for FilmArray examination, in which 140 patients were clinically diagnosed with suspected pertussis. The study was performed at SCMC, a tertiary teaching Children's hospital located in Shanghai, China, with complete sub-specialties. There are 650 beds and approximately 1.75 million patients annually, about $60-70 \%$ of whom were referred from other health care institutions. In that study, the positive rate of B. pertussis detected by FilmArray RP is $6.3 \%$ (49/775) in the whole setting and $35 \%(49 / 140)$ in pertussis suspected patients, but the clinical significance remained for further investigation (36). Therefore, the present study aims to investigate the clinical features of pertussis patients compared to patients with pertussis-like syndrome and to explore the possible clinical significance of pertussis coinfection.

\section{Methods}

\section{Study design and subjects}

The study population was enrolled according to the protocol definitions and inclusion criteria. A total of 851 patients with RTIs were screened in a previous study, and 775 eligible patients were enrolled according to the inclusion criteria (38). Among 775 patients, 140 were suspected of having pertussis according to the CDC definition: (I) cough for more than 2 weeks; and (II) paroxysmal cough, or inspiratory whoop, or post-tussive vomiting. In the present study, patients suspected of having pertussis were diagnosed with pertussis when $B$. pertussis was positive by FilmArray RP, and patients were otherwise diagnosed with pertussis-like syndrome.

The patients were assessed by the attending and chief physicians from the Department of Respiratory Medicine and the Department of Infectious Diseases. All of these physicians regularly received training on the clinical diagnosis of pertussis, which was based on both the medical history as well as personal observations on the day of hospitalization. The symptomatology was confirmed at least by two physicians from the Department of Respiratory Medicine and/or the Department of Infectious Diseases.

\section{Specimens}

Nasopharyngeal swab (NPS) or sputum specimens were obtained from patients with suspected pertussis on the day of hospitalization at SCMC from December 01, 2016 to November 30, 2017. Demographic information (i.e., age at enrollment and gender), medical history including vaccination history for pertussis as well as information of clinical symptoms and treatments received were documented for each enrolled patient. The study was approved by the Institutional Review Board and the Ethics Committee of SCMC (SCMCIRB-K2017044), and written informed consent was obtained from the parents of each patient.

\section{FilmArray RP v1.7 testing}

The FilmArray RP v1.7 targets 19 pathogens, including ADV, influenza A viruses H1, 2009H1, H3 (FluA-H1, FluA2009H1, FluA-H3) and FluB, parainfluenza virus types 1 to 4 (Para 1-4), coronaviruses 229E, HKU1, OC43, and NL63 (Cov-HKU1, NL63, 229E, OC43), human metapneumovirus (hMPV), RSV, Rhino/Entero, Chlamydophila pneumoniae (C. pneumoniae), Mycoplasma pneumoniae (M. pneumoniae) and $B$. pertussis. The principle of the FilmArray RP assay has been previously described $(32,36,37)$, and was performed according to the manufacturer's instructions.

\section{Statistical analysis}

SPSS software package v21.0 was used for all statistical analyses. Categorical variables were expressed as frequencies and percentages. The chi-square and Fisher's exact tests were used to compare groups. Continuous variables are expressed as the mean \pm standard deviation. Student's $t$-test was used to assess the statistical significance between groups. $\mathrm{P}<0.05$ was considered to be statistically significant.

\section{Results}

\section{Clinical characteristics of the patients}

The present study was a sub-group analysis of our previous study (38), and the study design is illustrated in Figure 1. A total of 140 patients who were suspected of having pertussis 


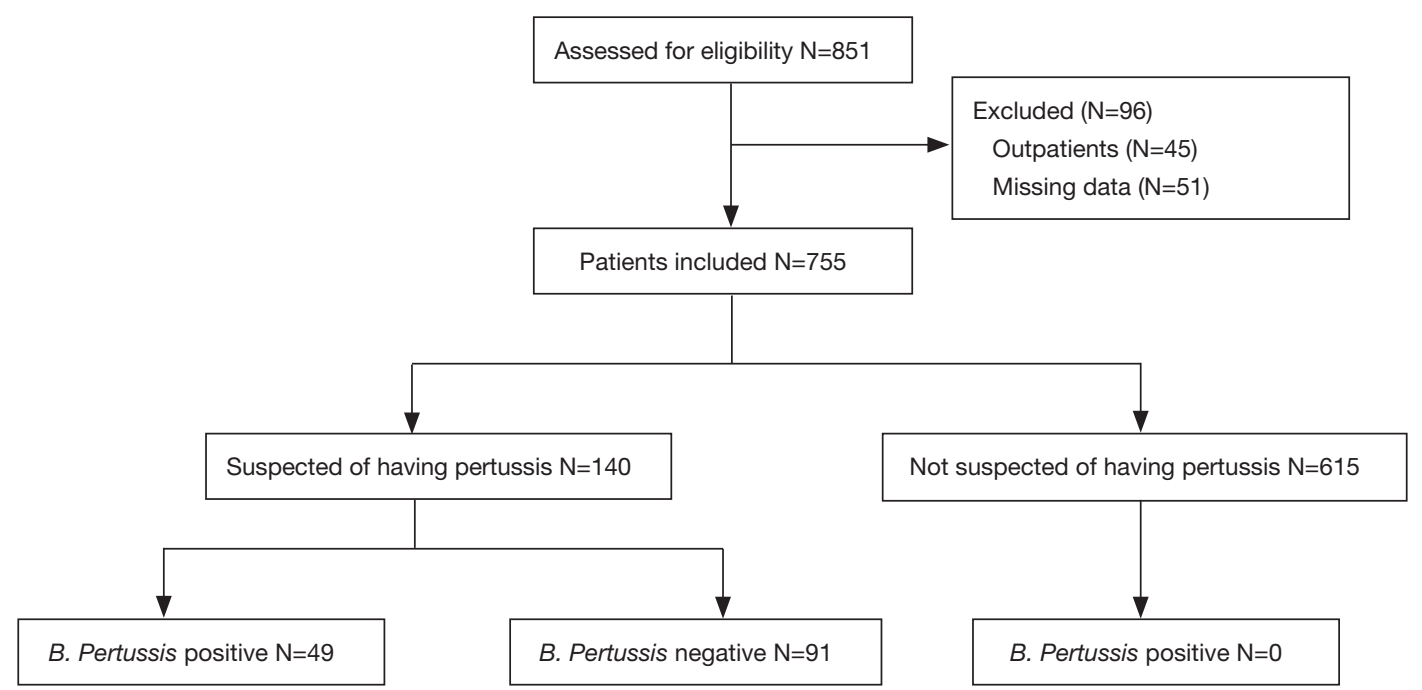

Figure 1 Flow diagram illustrating the design of the present study.

were enrolled in the present study between December 01, 2016, and November 30, 2017. Pertussis infection was confirmed in $35.0 \%(49 / 140)$ of the patients based on B. pertussis positive by FilmArray RP analysis, and the other $65.0 \%(91 / 140)$ of patients were diagnosed with pertussislike syndrome. Congenital heart disease was the most frequently observed underlying disease in these patients. The general characteristics of the enrolled patients, such as the contact history and vaccination status, are presented in Table 1 .

\section{Respiratory pathogens detected in patients with suspected pertussis}

Among the 140 specimens, 50.0\% (70/140) had a single pathogen, $45.0 \%(63 / 140)$ had multiple pathogens, and $5.0 \%(7 / 140)$ had no pathogens (Figure $2 A)$. The overall positivity rate of the specimens was $95.0 \%(133 / 140) . B$. pertussis was the most prevalent pathogen $(35.0 \%, 49 / 140)$, followed by Rhino/Entero $(32.9 \%, 46 / 140)$ and RSV $(31.4 \%, 44 / 140)$. The positivity rates of the other pathogens are presented in Figure $2 B$.

Forty-nine patients were positive for pertussis, among whom $35(71.4 \%)$ were co-detected with at least one virus or atypical bacteria (Figure $3 A$ ). A co-infection of B. pertussis with Rhino/Entero, RSV or Rhino/Entero + Para3 was most common and was detected in 8 (16.3\%), 5 (10.2\%) and $5(10.2 \%)$ patients, respectively. Up to four different pathogens were detected in a single sample. The multipathogen combinations are listed in Figure $3 A$ and Table $S 1$.
Ninety-one patients were detected pertussis negative, among whom $56(61.5 \%)$ were detected single-pathogen positive, $28(30.8 \%)$ were multi-pathogen positive and 7 (7.7\%) were negative. RSV, Para and Rhino/Entero are the most prevalent pathogens in the patients with pertussislike syndrome (Figure 3B).

\section{Clinical characteristics of the patients with pertussis and pertussis-like syndrome}

Regarding the clinical symptoms, there were no significant differences between the groups of patients with pertussis and pertussis-like syndrome (Table 2). However, more pertussis patients than pertussis-like syndrome patients were diagnosed at less than 6 months of age $\left(\chi^{2}=4.615, \mathrm{P}<0.05\right)$, and the number of patients with respiratory failure was higher in the pertussis-like syndrome group than in the pertussis group $\left(\chi^{2}=5.832, \mathrm{P}<0.05\right)$. Laboratory tests showed that the median number of white blood cells (WBCs) in the pertussis group was $18.40(13.05,23.69) \times 10^{9} / \mathrm{L}$, which was significantly higher than that in the pertussis-like syndrome group $(\mathrm{Z}=-6.254, \mathrm{P}<0.01)$. Moreover, the patients with pertussis were more likely to be co-infected with virus than the patients with pertussis-like syndrome $\left(\chi^{2}=22.509, \mathrm{P}<0.01\right)$.

\section{Clinical characteristics of patients with only pertussis and with pertussis/virus co-infections}

Among the 49 patients who tested positive for pertussis, $14(28.6 \%)$ patients were positive for only pertussis, 34 
Table 1 General characteristics of the patients enrolled

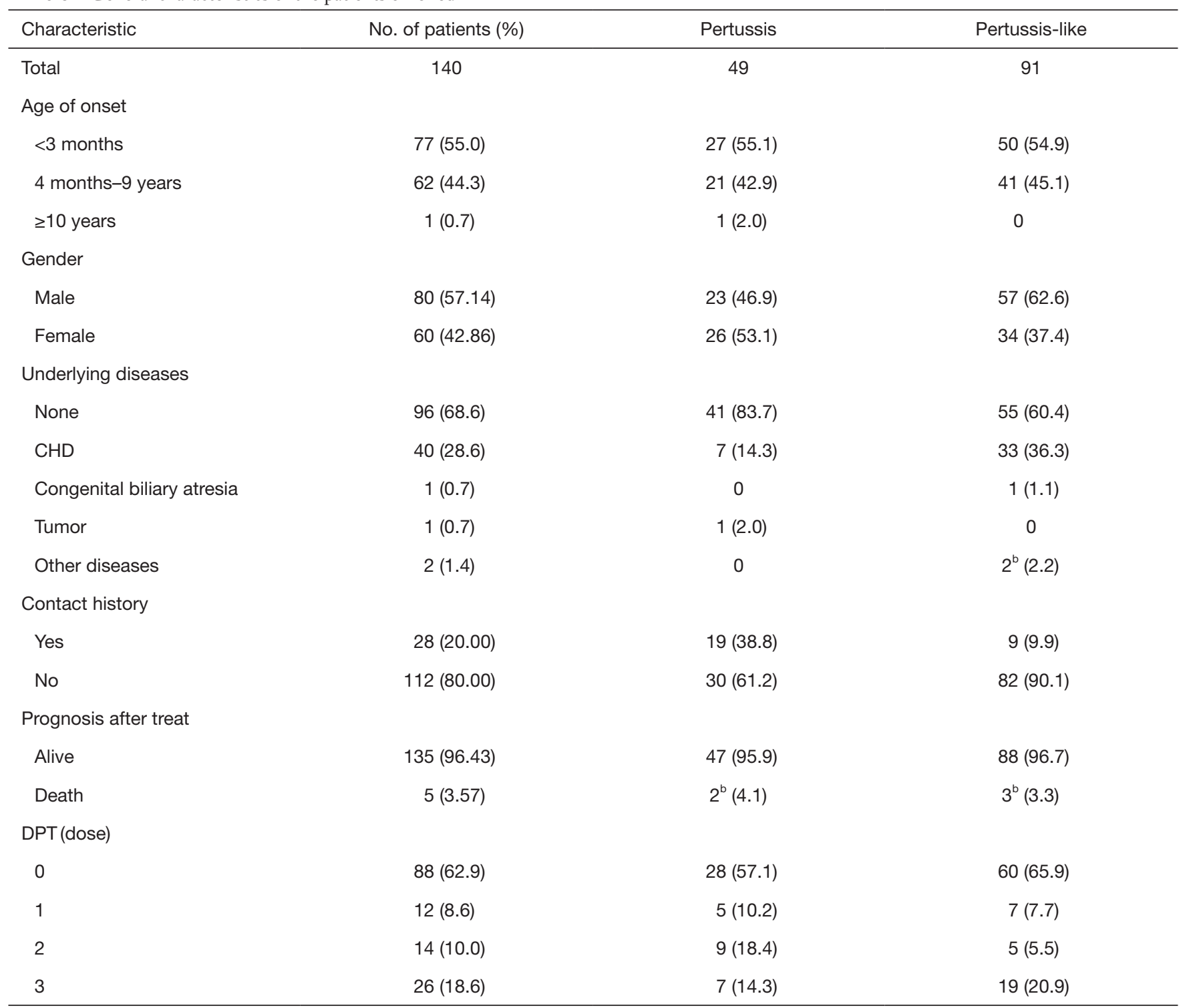

${ }^{a}$, other underlying diseases included cerebral dysplasia and congenital intestinal malrotation; ${ }^{b}$, cause of death: the two patients in the pertussis group died of neuroblastoma and hyperleukocytosis; two of the three patients in the pertussis-like group died of congenital heart disease and one of congenital intestinal malrotation. CHD, congenital heart disease; DPT, diphtheria, tetanus and pertussis; No., number.

(69.4\%) patients were co-detected with at least one virus, while 1 patient was co-detected with pertussis and $M$. pneumoniae. The most prevalent co-detected viruses were Rhino/Entero, RSV and Para 3. The clinical characteristics of patients with only pertussis and with pertussis/virus coinfections were evaluated, and there was no significant difference regarding the clinical symptoms or the laboratory data between these patient groups (Table 3). However, it is worth noting that $20.6 \%(7 / 34)$ of the patients with pertussis/virus co-infections were empirically treated with sulfamethoxazole-trimethoprim (SMZ-TMP), suggesting that these patients may be clinically considered resistant to microlides, although this conclusion cannot be confirmed due to the lack of pertussis culture and susceptibility testing. In addition, $100 \%$ of the patients with pertussis/ RSV co-infection experienced fever, which was significantly higher than the number of patients with only pertussis infection who also experienced fever. More patients in the pertussis/RSV group required respiratory support than in the pertussis-only group ( $75 \%$ vs. $28.6 \%$, respectively), 
A

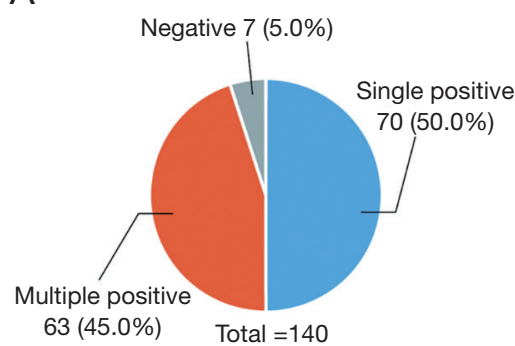

B

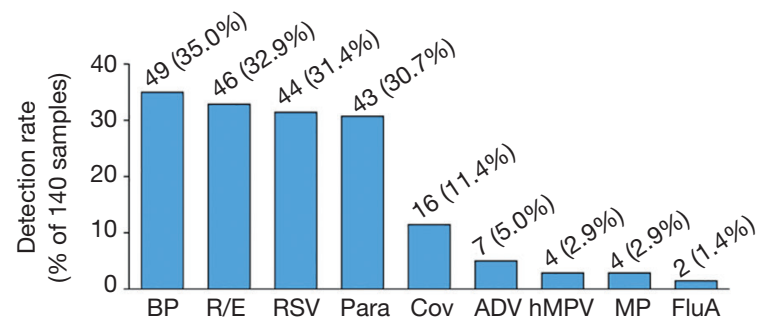

Figure 2 Overall detection of respiratory pathogens in 140 children with suspected pertussis. (A) The overall detection rates of the samples with single pathogen, multiple pathogens and no pathogen; (B) the positive rate of each respiratory pathogen. BP, B. pertussis; R/E, rhinovirus/enterovirus; RSV, respiratory syncytial virus; Para, parainfluenza virus; Cov, coronavirus; ADV, adenovirus; hMPV, human metapneumovirus; MP, M. pneumoniae; FluA, Influenza A virus.

A

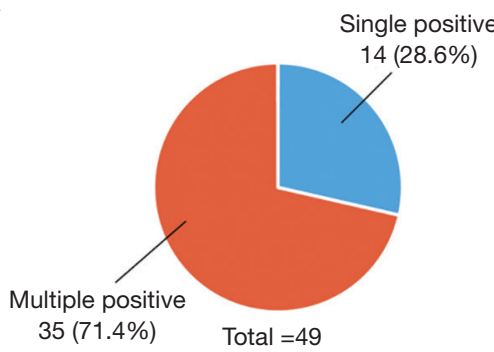

B

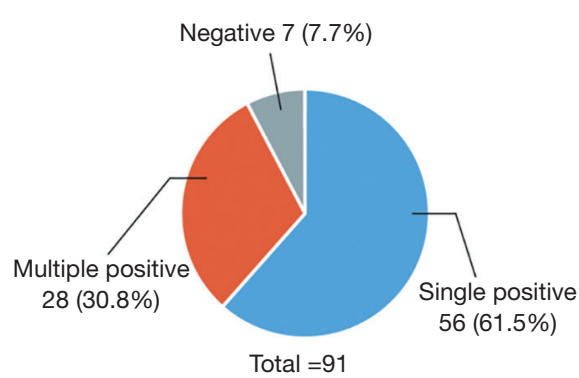

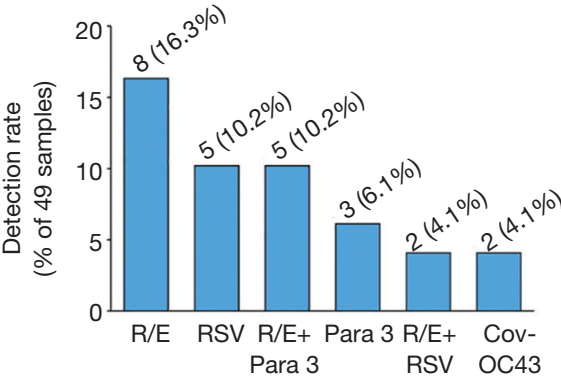

co-detected with $B$. pertussis

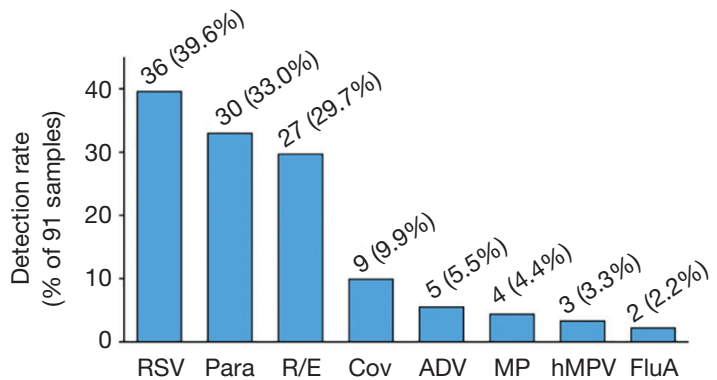

Figure 3 Detection of respiratory pathogens in children with pertussis and pertussis-like syndrome. (A) The detection rates of the samples with single pathogen and multiple pathogens in 49 children with pertussis, and the detection rates of multiple respiratory pathogens most commonly detected in these samples; (B) the detection rates of the samples with single positive samples, multiple positive samples and no pathogen in 91 children with pertussis-like syndrome, and the detection rate of each respiratory pathogen. R/E, rhinovirus/enterovirus; RSV, respiratory syncytial virus; Para, parainfluenza virus; Para3, parainfluenza virus type 3; Cov, coronavirus; Cov-OC43, coronavirus OC43; ADV, adenovirus; hMPV, human metapneumovirus; MP, M. pneumoniae; FluA, Influenza A virus.

although this difference was not significant (Table 4).

\section{Clinical characteristics of the patients with or without respiratory support}

A portion of the patients with pertussis in the study cohort
$(13 / 49,26.5 \%)$ required at least one form of respiratory support. Patients born prematurely tended to require oxygen intake more frequently than other patients, which was similar to those in the unvaccinated group. A total of $23.1 \%$ of the patients (3/13) who needed respiratory support had an apnea history, and the hospitalization time for this 
Table 2 Comparison of the clinical characteristics of children with pertussis and pertussis-like syndrome

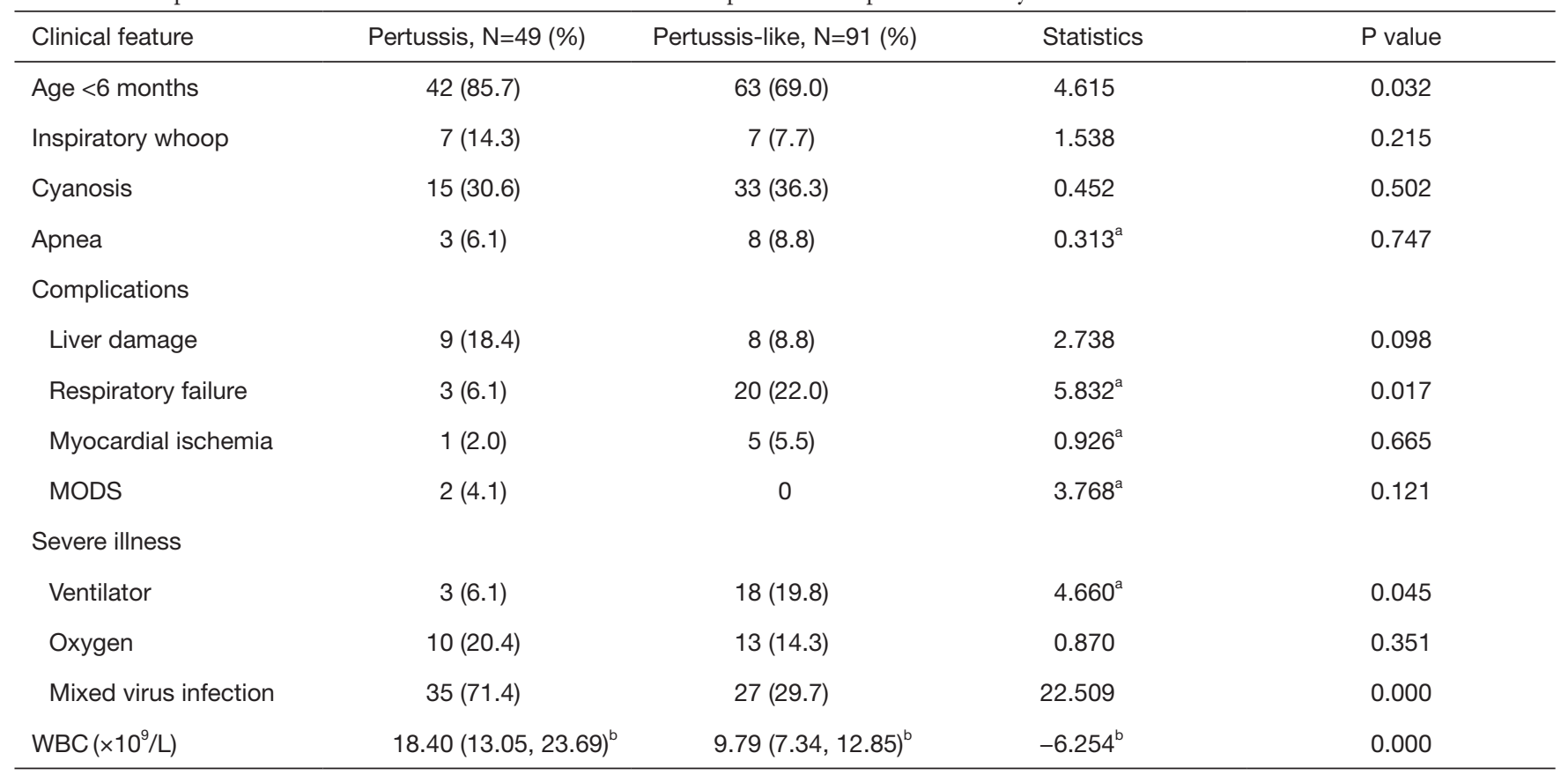

${ }^{\mathrm{a}}$, Fisher's exact test; ${ }^{\mathrm{b}}$, data lacking a normal distribution are expressed as the median and quartiles, i.e., median (25\%, $\left.75 \%\right)$, and the statistic is $Z$ value. MODS, multiple organ dysfunction syndrome; WBC, white blood cell.

Table 3 Comparison of the clinical characteristics of the patients with only pertussis and with pertussis/virus co-infections

\begin{tabular}{|c|c|c|c|c|}
\hline Clinical feature & Single BP, N=14 (\%) & Mixed BP/viruses, $\mathrm{N}=34$ (\%) & Statistics & $P$ value \\
\hline Fever & $4(28.6)$ & $6(17.6)$ & $0.718^{\mathrm{a}}$ & 0.448 \\
\hline Wheeze & $4(28.6)$ & $16(47.1)$ & $1.394^{\mathrm{a}}$ & 0.338 \\
\hline Cyanosis & $5(35.7)$ & $10(29.4)$ & 0.183 & 0.669 \\
\hline Apnea & $1(7.1)$ & $2(5.9)$ & $0.027^{\mathrm{a}}$ & 1.000 \\
\hline CHD & $3(21.4)$ & $4(11.8)$ & $0.743^{\mathrm{a}}$ & 0.400 \\
\hline Time of hospitalization (d) & $10.29 \pm 6.17$ & $8.86 \pm 4.85$ & 0.964 & 0.340 \\
\hline WBC $\left(\times 10^{9} / L\right)$ & $16.98(12.06,23.26)^{b}$ & $18.72(13.27,24.70)^{b}$ & $-0.953^{b}$ & 0.341 \\
\hline LY\% & $65.25(56.75,70.38)^{\mathrm{b}}$ & $71.05(62.60,76.15)^{b}$ & $-1.837^{b}$ & 0.066 \\
\hline
\end{tabular}

a , Fisher's exact test; ${ }^{b}$, data lacking a normal distribution are expressed as the median and quartiles, i.e., median (25\%, 75\%), and the statistic is Z value. BP, B. pertussis; CHD, congenital heart disease; LY, lymphocyte; SMZ, sulfamethoxazole; TMP, trimethoprim; WBC, white blood cell. 
Table 4 Comparison of the clinical characteristics of patients with only pertussis and with pertussis/RSV co-infection

\begin{tabular}{|c|c|c|c|c|}
\hline Clinical feature & Single BP, N=14, (\%) & Mixed BP/RSV, N=8, (\%) & Statistics & $P$ value \\
\hline Fever & $4(28.6)$ & $8(100.0)$ & $10.476^{a}$ & 0.002 \\
\hline Wheeze & $4(28.6)$ & $6(75.0)$ & $4.426^{a}$ & 0.074 \\
\hline Inspiratory whoop & $4(28.6)$ & 0 & $2.794^{a}$ & 0.254 \\
\hline Apnea & $1(7.1)$ & $1(12.5)$ & $0.177^{\mathrm{a}}$ & 1.000 \\
\hline $\mathrm{CHD}$ & $3(21.4)$ & $1(12.5)$ & $0.273^{\mathrm{a}}$ & 1.000 \\
\hline Complications & $2(14.3)$ & $4(50.0)$ & $3.274^{a}$ & 0.137 \\
\hline Need of oxygen & $4(28.6)$ & $6(75.0)$ & $4.426^{\mathrm{a}}$ & 0.074 \\
\hline WBC $\left(\times 10^{9} / L\right)$ & $16.98(12.06,23.26)^{b}$ & $18.72(13.27,24.70)^{b}$ & $-1.160^{b}$ & 0.267 \\
\hline LY\% & $62.79 \pm 16.63$ & $51.75 \pm 16.57$ & 0.838 & 0.412 \\
\hline
\end{tabular}

${ }^{a}$, Fisher's exact test; ${ }^{b}$, data lacking a normal distribution are expressed as the median and quartiles, i.e., median (25\%, 75\%), and the statistic is Z value. BP, B. pertussis; CHD, congenital heart disease; LY, lymphocyte; RSV, respiratory syncytial virus; SMZ, sulfamethoxazole; TMP, trimethoprim; WBC, white blood cell.

group was significantly longer than that of those without respiratory support (14vs. $6 \mathrm{~d}$, respectively). The initial WBC counts of these two groups were comparable, but the lymphocyte ratio was lower in the patients with respiratory support than in those without respiratory support (Table 5).

\section{Treatment and prognosis of patients with pertussis}

The 49 patients with pertussis were treated with azithromycin or erythromycin together with symptomatic treatment. Seven $(14.3 \%)$ patients who were clinically confirmed as erythromycin-resistant were switched to SMZ-TMP treatment, among whom 6 patients were discharged after fully recovering, and 1 patient died of hyperleukocytosis combined with pulmonary hypertension and multiple organ failure. Among the 42 treatmentsensitive patients, one patient died of neuroblastoma combined with immunodeficiency. Both of these patients were unvaccinated. For the 91 patients with pertussislike syndrome, in addition to antiviral and symptomatic treatment, appropriate antibiotics were also prescribed according to the results of sputum culture. Eighty-eight (96.7\%) patients were discharged after fully recovering, and 3 patients died ( 2 of congenital heart disease and 1 of intestinal malrotation).

\section{Discussion}

In the present study, 140 specimens were collected from pediatric patients with suspected pertussis. B. pertussis was the most prevalent pathogen $(35.0 \%, 49 / 140)$, followed by Rhino/Entero (32.9\%, 46/140) and RSV (31.4\%, 44/140). More patients were diagnosed with pertussis than with pertussis-like syndrome at less than 6 months of age, and pertussis patients had higher WBC counts and were more likely to be co-infected with viruses than patients with pertussis-like syndrome. The patients with pertussis/virus co-infections were more likely to be empirically treated with SMZ-TMP. More patients in the pertussis/RSV group than in the pertussis-only group required respiratory support. In addition, the premature and unvaccinated groups tended to require oxygen intake more frequently than the other groups.

The major limitation of the two reference standards for pertussis that are currently used in China $(38,39)$ is the lack of positive PCR results as the criteria for laboratory diagnosis. Multiple studies pointed out that this limitation together with a lack of other laboratory diagnostic methods, insufficient clinical experience with atypical cases and low awareness of the disease, resulted in the underestimation of the incidence of pertussis infection in China (23,40-42). In 2017, a 
Table 5 Comparison of the clinical characteristics of the 49-pertussis positive patients with or without respiratory support

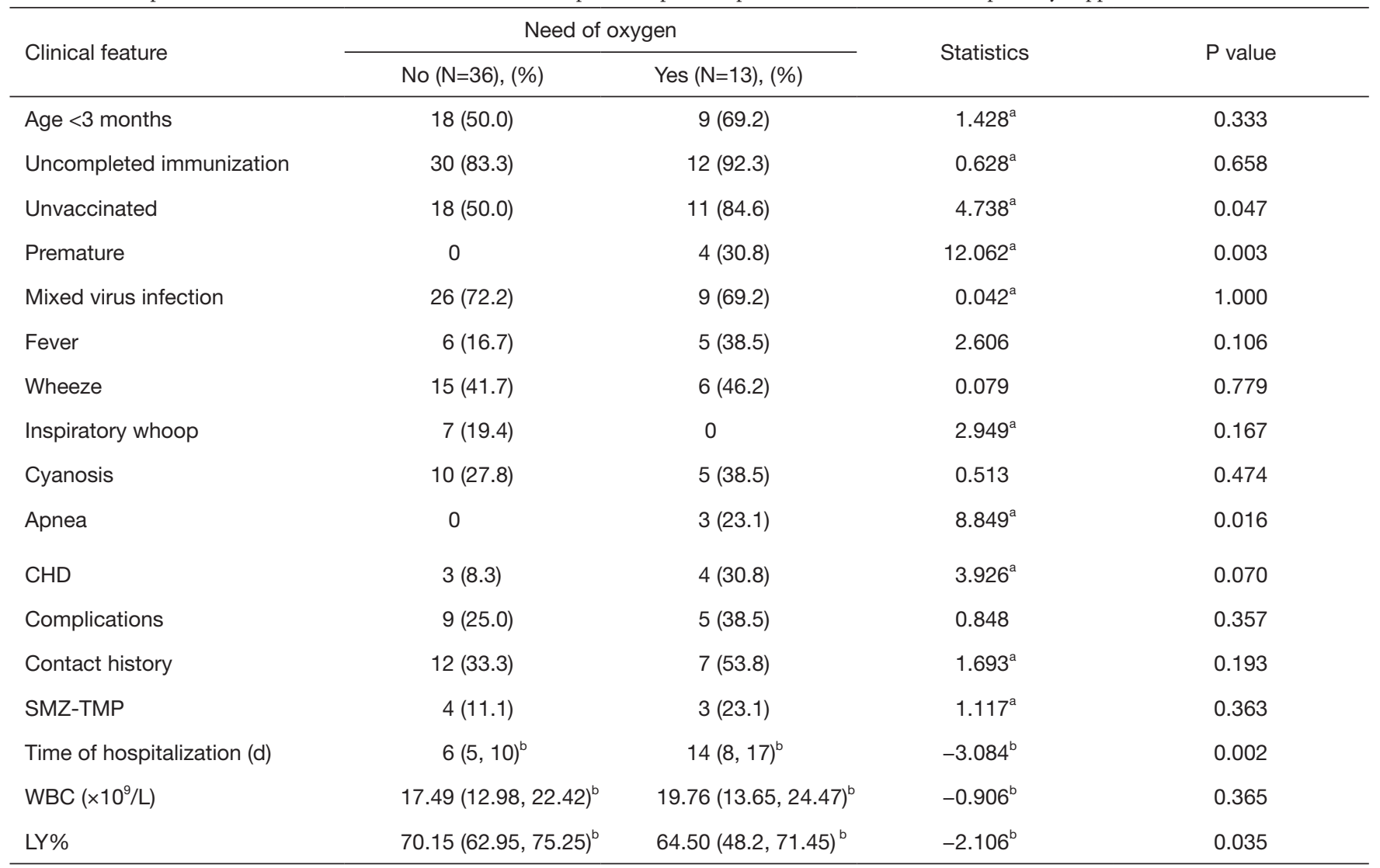

a, Fisher's exact test; ${ }^{b}$, data lacking a normal distribution are expressed as the median and quartiles, i.e., median (25\%, 75\%), and the statistic is $\mathrm{Z}$ value. BP, B. pertussis; CHD, congenital heart disease; LY, lymphocyte; RSV, respiratory syncytial virus; SMZ, sulfamethoxazole; TMP, trimethoprim; WBC, white blood cell.

new version of "Diagnosis and treatment recommendations in Chinese pediatric pertussis patients" was published, in which both clinical and laboratory diagnostic criteria for agespecific pertussis were modified according to the clinical case definitions for pertussis proposed by the Global Pertussis Initiative (GPI) $(43,44)$. In our study, all 49 pertussis patients met the clinical and laboratory diagnostic criteria of the new recommendations. However, it is worth mentioning that the median WBC count in patients less than 3 months old was only $17.33 \times 10^{9} / \mathrm{L}$, which was lower than the laboratory diagnostic criteria (WBC counts $\geq 20 \times 10^{9} / \mathrm{L}$ ), indicating that clinicians should pay attention to the risk of pertussis infection in infants with relatively low WBC counts.

In China, routine vaccination against pertussis with DTwP (a whole-cell pertussis vaccine combined with diphtheria and tetanus toxoids) was introduced in the early 1960s, when 3 doses of DTwP were given at 3, 4 and
5 months of age. In 1982, a booster dose at 18-24 months of age was added (45). Since 2007, the DTwP vaccine has been replaced by the DTaP (acellular pertussis vaccine combined with diphtheria and tetanus) vaccine (46). The $\mathrm{DTaP}$ vaccine is much safer than the $\mathrm{DTw} P$ vaccine, but the immunization duration of the former is only 5-6 years. A previous study showed that for small infants, the effective rate can reach $91.7 \%$ after inoculation with a 3 -dose DTP (diphtheria-tetanus toxoids-pertussis) vaccine, but can reach only $46 \%$ with a 1 -dose vaccination (47). In our study, 42 of the 49 patients $(85.7 \%)$ with pertussis did not complete the entire immunization process, which might have contributed to the high prevalence rate. The unvaccinated group required respiratory support more frequently, indicating that even incomplete immunization was beneficial to the patients. The positivity rate of pertussis after inoculation with the 1-, 2- and 3-dose DTaP vaccines (10.20\%, 18.37\% and $14.29 \%$, respectively) showed no significant differences, 
and 19 of the 49 patients (38.8\%) with pertussis had a confirmed contact history with family members who had a cough. These results indicate that atypical cases may be responsible for ongoing transmission. Therefore, in addition to the three basic immunizations and one booster immunization of the pertussis vaccine, the 2015 WHO recommendation suggested administering the $\mathrm{DTaP}$ vaccine to children $>7$ years of age, adolescents and pregnant women (48).

Macrolide antibiotics are the first choice for pertussis treatment, and the treatment timing has direct effects on the prognosis and outcome. The use of macrolide antibiotics in the catarrhal stage can reduce or even prevent coughing. In the paroxysmal stage, the course of the disease cannot be shortened by the application of sensitive antibiotics; however, antibiotic use can effectively shorten the sterilization period, killing the flora attached to the nasopharynx, limiting the spread of the pathogen and preventing secondary infections. In our study, one patient was treated with cephalosporin for 2 weeks for a "RTI", and the WBC counts became progressively elevated (from $8.4 \times 10^{9} / \mathrm{L}$ to $134.73 \times 10^{9} / \mathrm{L}$ ). The patient was then transferred to our hospital for suspected leukemia but was soon diagnosed with a $B$. pertussis, Rhino/Entero and RSV co-infection. Moreover, the patient was diagnosed with severe pulmonary hypertension, which may have been caused by the luminal aggregates of abundant leukocytes in small pulmonary arteries, veins and lymphatics (49). Although symptomatic treatments including anti-infective therapy, mechanical ventilation and exchange transfusion were carried out, the patient still died of multiple organ failure. A previous study showed that the use of exchange transfusion to reduce WBC counts can significantly reduce the release of the pertussis toxin (PT) and can improve the mortality of pertussis hypoxemia, but it is better for this treatment being performed before cardiogenic shock or organ failure (50). On the other hand, there were 7 other patients suspected of having pertussis combined with hypoxemia with $\mathrm{WBC}$ counts $>20 \times 10^{9} / \mathrm{L}$ by the end of this study. All patients were diagnosed with $B$. pertussis infection during the early stage of the disease, followed by macrolide treatment and ventilator-assisted ventilation. Four cases with WBC counts $>50 \times 10^{9} / \mathrm{L}$ underwent exchange transfusion, and all 7 patients were clinically improved and discharged. Therefore, our results strongly suggest that early diagnosis and macrolide treatment play important roles in the treatment and prognosis of pertussis.

Previous studies have shown that pertussis infection in children sometimes mimics viral RTIs, especially in infants $(6,27,35)$. In our study, there were no significant differences regarding the clinical symptoms between the two groups. RSV, Para and Rhino/Entero are the most prevalent causative pathogens in patients with pertussis-like syndrome that can have similar clinical presentations. These results again suggest that distinguishing pertussis from pertussislike syndrome based on clinical symptoms is not feasible, and emphasize the importance of laboratory tests as an indispensable complementary method in the clinical setting.

The co-infection of $B$. pertussis with respiratory viruses has been reported previously. However, the clinical relevance of such co-infections is still under investigation. In our study, no significant differences were observed regarding the clinical symptoms and laboratory data in patients with only pertussis and patient with pertussis/virus co-infections. When more attention was paid to the coinfection of pertussis with Rhino/Entero, RSV or Para 3, the clinical data indicated that pertussis/RSV co-infection tended to be more severe (Table 4), while no significant differences were observed in pertussis-Rhino/Entero or pertussis-Para 3 co-infection compared to pertussis-only infections (data not shown). These results raised another long-standing unresolved question: how can "real" causative pathogens be identified in multi-pathogen positive patients, especially when the results are obtained by molecular approaches? Under such circumstances, the quantification of the mRNA levels of the pathogen at multiple time points during the entire disease course might be helpful to distinguish the "real" causative pathogen.

At present, most of the PCR-based assays, either laboratory or commercially developed, target the insertion sequence (IS481) for B. pertussis detection (51-54). IS481 is internally repeated $>50$ times in each $B$. pertussis genome, resulting in high analytical sensitivity. However, studies have shown the presence of IS481 in Bordetella bolmesii ( $B$. bolmesii), an microorganism that is also associated with an illness with a cough $(55,56)$. The genetic target for $B$. pertussis in the FilmArray RP, however, is the promoter region of the $\mathrm{PT}$ gene, and there is only one copy in each $B$. pertussis genome (32). This target is specific, with no cross-reactivity with $B$. holmesii, but may have lower sensitivity than the IS481 locus. Two studies showed discrepant conclusions regarding the sensitivity of targeting the PT gene. While Jerris et al. found a loss of analytical sensitivity for B. pertussis using the FilmArray RP, Qin et al. demonstrated that there was little difference between the detection limit for the PT gene and IS481 $(51,54)$. In our 
study, 49 out of 140 patients were shown to be $B$. pertussispositive using the FilmArray RP. However, due to the lack of other laboratory tests at our center during the study period, the evaluation of the sensitivity of the FilmArray RP by targeting IS481 or other loci was not performed.

Our study also has several limitations. First, this study was performed at a single center, and the sample size was small. Second, we did not have data from an appropriate assay to evaluate the sensitivity and specificity of the FilmArray RP, and B. parapertussis could not be detected in the current assay. Additionally, pertussis culture and susceptibility tests were not available during the study period.

\section{Conclusions}

As in other developing countries, epidemiologic surveillance for B. pertussis is essential in China, especially in the children who could benefit most from the vaccine. The present study describes the current status of $B$. pertussis infection in China and highlights the need for timely and accurate diagnosis based on both clinical symptoms and laboratory methods.

\section{Acknowledgments}

We would like to thank the patients and their parents for the support and cooperation in publishing this work.

Funding: This work was financially supported by the Natural Science Foundation of Shanghai (16ZR1421600), Medical-Engineering Cross Fund of Shanghai Jiao Tong University (YG2016QN38), the Collaborative Innovation Center for Translational Medicine at Shanghai Jiao Tong University School of Medicine (TM201616), and the Love Charity Foundation Research Project in Shanghai Children's Medical Center (2017SCMC-AY004).

\section{Footnote}

Conflicts of Interest: $\mathrm{Y} \mathrm{Xia}$ is an employee of bioMérieux. The other authors have no conflicts of interest to declare.

Ethical Statement: The authors are accountable for all aspects of the work in ensuring that questions related to the accuracy or integrity of any part of the work are appropriately investigated and resolved. The study was approved by the Institutional Review Board and the Ethics Committee of Shanghai Children's Medical Center (SCMCIRB-K2017044), and written informed consent was obtained from the parents of each patient.

\section{References}

1. Edwards KM. Overview of pertussis: focus on epidemiology, sources of infection, and long term protection after infant vaccination. Pediatr Infect Dis J 2005;24:S104-8.

2. Elam-Evans LD, Yankey D, Singleton JA, et al. National, state, and selected local area vaccination coverage among children aged 19-35 months - United States, 2013. MMWR Morb Mortal Wkly Rep 2014;63:741-8.

3. Seither R, Masalovich S, Knighton CL, et al. Vaccination coverage among children in kindergarten - United States, 2013-14 school year. MMWR Morb Mortal Wkly Rep 2014;63:913-20.

4. Haghighi F, Shahcheraghi F, Abbasi E, et al. Genetic Profile Variation in Vaccine Strains and Clinical Isolates of Bordetella pertussis Recovered from Iranian Patients. Avicenna J Med Biotechnol 2014;6:178-84.

5. Shojaei J, Saffar M, Hashemi A, et al. Clinical and laboratory features of pertussis in hospitalized infants with confirmed versus probable pertussis cases. Ann Med Health Sci Res 2014;4:910-4.

6. van den Brink G, Wishaupt JO, Douma JC, et al. Bordetella pertussis: an underreported pathogen in pediatric respiratory infections, a prospective cohort study. BMC Infect Dis 2014;14:526.

7. Guris D, Strebel PM, Bardenheier B, et al. Changing epidemiology of pertussis in the United States: increasing reported incidence among adolescents and adults, 19901996. Clin Infect Dis 1999;28:1230-7.

8. de Melker HE, Schellekens JF, Neppelenbroek SE, et al. Reemergence of pertussis in the highly vaccinated population of the Netherlands: observations on surveillance data. Emerg Infect Dis 2000;6:348-57.

9. Godfroid F, Denoel P, Poolman J. Are vaccination programs and isolate polymorphism linked to pertussis reemergence? Expert Rev Vaccines 2005;4:757-78.

10. Chiappini E, Stival A, Galli L, et al. Pertussis reemergence in the post-vaccination era. BMC Infect Dis 2013;13:151.

11. Zhang Q, Zheng H, Liu M, et al. The seroepidemiology of immunoglobulin $\mathrm{G}$ antibodies against pertussis toxin in China: a cross sectional study. BMC Infect Dis 2012;12:138.

12. Xu Y, Wang L, Xu J, et al. Seroprevalence of pertussis in China: need to improve vaccination strategies. Hum 
Vaccin Immunother 2014;10:192-8.

13. van der Zee A, Schellekens JF, Mooi FR. Laboratory Diagnosis of Pertussis. Clin Microbiol Rev 2015;28:1005-26.

14. de Melker HE, Versteegh FG, Conyn-Van Spaendonck MA, et al. Specificity and sensitivity of high levels of immunoglobulin $\mathrm{G}$ antibodies against pertussis toxin in a single serum sample for diagnosis of infection with Bordetella pertussis. J Clin Microbiol 2000;38:800-6.

15. Sheridan SL, Ware RS, Grimwood K, et al. Number and order of whole cell pertussis vaccines in infancy and disease protection. JAMA 2012;308:454-6.

16. Grogan JA, Logan C, O'Leary J, et al. Real-time PCRbased detection of Bordetella pertussis and Bordetella parapertussis in an Irish paediatric population. J Med Microbiol 2011;60:722-9.

17. Gao F, Mahoney JC, Daly ER, et al. Evaluation of a multitarget real-time PCR assay for detection of Bordetella species during a pertussis outbreak in New Hampshire in 2011. J Clin Microbiol 2014;52:302-6.

18. Holberg-Petersen M, Jenum PA, Mannsaker T, et al. Comparison of PCR with culture applied on nasopharyngeal and throat swab specimens for the detection of Bordetella pertussis. Scand J Infect Dis 2011;43:221-4.

19. Abu Raya B, Bamberger E, Gershtein R, et al. The laboratory diagnosis of Bordetella pertussis infection: a comparison of semi-nested PCR and real-time PCR with culture. Eur J Clin Microbiol Infect Dis 2012;31:619-22.

20. World Health Organization: WHO-recommended standards for surveillance of selected vaccine-preventable diseases. 2003.

21. Centers for Disease Control and Prevention (CDC): Manual for the Surveillance of Vaccine-Preventable Diseases: Chapter10: Pertussis. 2014.

22. Zhang L, Xu Y, Zhao J, et al. Effect of vaccination on Bordetella pertussis strains, China. Emerg Infect Dis 2010;16:1695-701.

23. Yao KH, Deng JK, Dawuti R. Pertussis diagnosis: the limitation of the currently used criteria and the suggestion of Global Pertussis Initiative. Zhongguo Dang Dai Er Ke Za Zhi 2016;18:891-6.

24. Cosnes-Lambe C, Raymond J, Chalumeau M, et al. Pertussis and respiratory syncytial virus infections. Eur J Pediatr 2008;167:1017-9.

25. Nuolivirta K, Koponen P, He Q, et al. Bordetella pertussis infection is common in nonvaccinated infants admitted for bronchiolitis. Pediatr Infect Dis J 2010;29:1013-5.
26. Abu Raya B, Bamberger E, Kassis I, et al. Bordetella pertussis infection attenuates clinical course of acute bronchiolitis. Pediatr Infect Dis J 2013;32:619-21.

27. Pavic-Espinoza I, Bendezu-Medina S, Herrera-Alzamora A, et al. High prevalence of Bordetella pertussis in children under 5 years old hospitalized with acute respiratory infections in Lima, Peru. BMC Infect Dis 2015;15:554.

28. Dube FS, Kaba M, Robberts FJ, et al. Respiratory microbes present in the nasopharynx of children hospitalised with suspected pulmonary tuberculosis in Cape Town, South Africa. BMC Infect Dis 2016;16:597.

29. McDonough EA, Barrozo CP, Russell KL, et al. A multiplex PCR for detection of Mycoplasma pneumoniae, Chlamydophila pneumoniae, Legionella pneumophila, and Bordetella pertussis in clinical specimens. Mol Cell Probes 2005;19:314-22.

30. van Kruijssen AM, Templeton KE, van der Plas RN, et al. Detection of respiratory pathogens by real-time PCR in children with clinical suspicion of pertussis. Eur J Pediatr 2007;166:1189-91.

31. Kumar S, Wang L, Fan J, et al. Detection of 11 common viral and bacterial pathogens causing communityacquired pneumonia or sepsis in asymptomatic patients by using a multiplex reverse transcription-PCR assay with manual (enzyme hybridization) or automated (electronic microarray) detection. J Clin Microbiol 2008;46:3063-72.

32. Poritz MA, Blaschke AJ, Byington CL, et al. FilmArray, an automated nested multiplex PCR system for multipathogen detection: development and application to respiratory tract infection. PLoS One 2011;6:e26047.

33. Qin X, Zerr DM, Kronman MP, et al. Comparison of molecular detection methods for pertussis in children during a state-wide outbreak. Ann Clin Microbiol Antimicrob 2016;15:28.

34. Huang H, Deng L, Xiao F, et al. Clinical analysis of children with pertussis and significance of respiratory virus detection in the combined diagnosis. Zhonghua Er Ke Za Zhi 2017;55:580-5.

35. Mahmoudi S, Banar M, Pourakbari B, et al. Identification of Etiologic Agents of the Pertussis-like Syndrome in Children by Real-time PCR Method. Prague Med Rep 2018;119:61-9.

36. Li J, Tao Y, Tang M, et al. Rapid detection of respiratory organisms with the FilmArray respiratory panel in a large children's hospital in China. BMC Infect Dis 2018;18:510.

37. Loeffelholz MJ, Pong DL, Pyles RB, et al. Comparison of the FilmArray Respiratory Panel and Prodesse real-time PCR assays for detection of respiratory pathogens. J Clin 
Microbiol 2011;49:4083-8.

38. National Health Commission of the People's Republic of China: Diagnostic criteria and principles of management of pertussis. 1995.

39. National Health Commission of the People's Republic of China: WS 274-2007 Diagnostic criteria for pertussis. 2007.

40. Chen Z, Zhang J, Cao L, et al. Seroprevalence of pertussis among adults in China where whole cell vaccines have been used for 50 years. J Infect 2016;73:38-44.

41. He H, Yao P, Zhou Y, et al. Is Pertussis Infection Neglected in China? Evidence from a Seroepidemiology Survey in Zhejiang, an Eastern Province of China. PLoS One 2016;11:e0155965.

42. Koh MT, Liu CS, Chiu CH, et al. Under-recognized pertussis in adults from Asian countries: a cross-sectional seroprevalence study in Malaysia, Taiwan and Thailand. Epidemiol Infect 2016;144:1192-200.

43. Cherry JD, Tan T, Wirsing von Konig CH, et al. Clinical definitions of pertussis: Summary of a Global Pertussis Initiative roundtable meeting, February 2011. Clin Infect Dis 2012;54:1756-64.

44. Deng J. Diagnosis and treatment recommendations in Chinese pediatric pertussis patients. Chin J Pediatr 2017;55:568-72.

45. World Health Organization. WHO vaccine preventable diseases: monitoring system. 2000 global summary. Geneva: The Organization, 2001.

46. Diao LD, Xu AQ, Chinese Prevention Medicine A. Consensus on the immunological defence of pertussis. Zhonghua Liu Xing Bing Xue Za Zhi 2011;32:550-3.

47. Bisgard KM, Rhodes P, Connelly BL, et al. Pertussis vaccine effectiveness among children 6 to 59 months

Cite this article as: Tao Y, Tang M, Luo L, Xiang L, Xia Y, Li B, Cao Q, Mo X. Identification of etiologic agents and clinical characteristics for patients suspected of having pertussis in a large Children's Hospital in China. Ann Transl Med 2019;7(18):443. doi: 10.21037/atm.2019.08.85 of age in the United States, 1998-2001. Pediatrics

2005;116:e285-94.

48. WHO. Pertussis vaccines: WHO position paper, August 2015--Recommendations. Vaccine 2016;34:1423-5.

49. Paddock CD, Sanden GN, Cherry JD, et al. Pathology and pathogenesis of fatal Bordetella pertussis infection in infants. Clin Infect Dis 2008;47:328-38.

50. Onoro G, Salido AG, Martinez IM, et al. Leukoreduction in patients with severe pertussis with hyperleukocytosis. Pediatr Infect Dis J 2012;31:873-6.

51. Qin X, Galanakis E, Martin ET, et al. Multitarget PCR for diagnosis of pertussis and its clinical implications. J Clin Microbiol 2007;45:506-11.

52. Lanotte P, Plouzeau C, Burucoa C, et al. Evaluation of four commercial real-time PCR assays for detection of Bordetella spp. in nasopharyngeal aspirates. J Clin Microbiol 2011;49:3943-6.

53. Hassan F, Hays L, Bell J, et al. Evaluation of 3 analytespecific reagents for detection of Bordetella pertussis and Bordetella parapertussis in clinical specimens. Diagn Microbiol Infect Dis 2014;80:181-4.

54. Jerris RC, Williams SR, MacDonald HJ, et al. Testing implications of varying targets for Bordetella pertussis: comparison of the FilmArray Respiratory Panel and the Focus B. pertussis PCR assay. J Clin Pathol 2015;68:394-6.

55. Loeffelholz MJ, Thompson CJ, Long KS, et al. Detection of Bordetella holmesii using Bordetella pertussis IS481 PCR assay. J Clin Microbiol 2000;38:467.

56. Bottero D, Griffith MM, Lara C, et al. Bordetella holmesii in children suspected of pertussis in Argentina. Epidemiol Infect 2013;141:714-7. 
Supplementary

Table S1 The multi-analyte-positive samples combined with $B$. pertussis

\begin{tabular}{|c|c|c|c|c|}
\hline Analyte 1 & Analyte 2 & Analyte 3 & Analyte 4 & $\begin{array}{c}\text { No. of multi-analyte positive } \\
\text { samples }\end{array}$ \\
\hline \multirow[t]{16}{*}{ B. pertussis } & $H / E$ & & & 8 \\
\hline & RSV & & & 5 \\
\hline & $\mathrm{H} / \mathrm{E}$ & Para 3 & & 5 \\
\hline & Para 3 & & & 3 \\
\hline & RSV & $\mathrm{H} / \mathrm{E}$ & & 2 \\
\hline & Cov-OC43 & & & 2 \\
\hline & ADV & & & 1 \\
\hline & $H / E$ & ADV & & 1 \\
\hline & hMPV & $H / E$ & & 1 \\
\hline & Para 3 & Para 4 & & 1 \\
\hline & RSV & Para 3 & & 1 \\
\hline & $H / E$ & Cov-HKU1 & & 1 \\
\hline & Cov-OC43 & Para 3 & & 1 \\
\hline & Cov-OC43 & Cov-229E & & 1 \\
\hline & Cov-OC43 & Cov-229E & Para 3 & 1 \\
\hline & Cov- HKU1 & $H / E$ & Para 3 & 1 \\
\hline
\end{tabular}

R/E, rhinovirus/enterovirus; RSV, respiratory syncytial virus; Para 3, parainfluenza virus type 3; Para 4, parainfluenza virus type 4; CovOC43, coronavirus OC43; Cov-HKU1, coronavirus HKU1; Cov-229E, coronavirus 229E; ADV, adenovirus; hMPV, human metapneumovirus. 